\title{
Durasol - Innover pour la durabilité des systèmes solaires
}

Une plateforme multi-sites pour étudier le vieillissement accéléré des composants et systèmes solaires photovoltaïques et thermiques et des corrélations climatiques via des plates-formes.

Ce projet a comme objectif la mise en place d'une plateforme de R\&D sur plusieurs sites, 9 à ciel ouvert et 5 à l'intérieur de laboratoires, pour étudier la durée de vie des trois technologies

définir des lois physiques de vieillissement des composants pour les trois technologies qui pourront également permettre de développer de futurs matériaux et procédés plus fiables. La

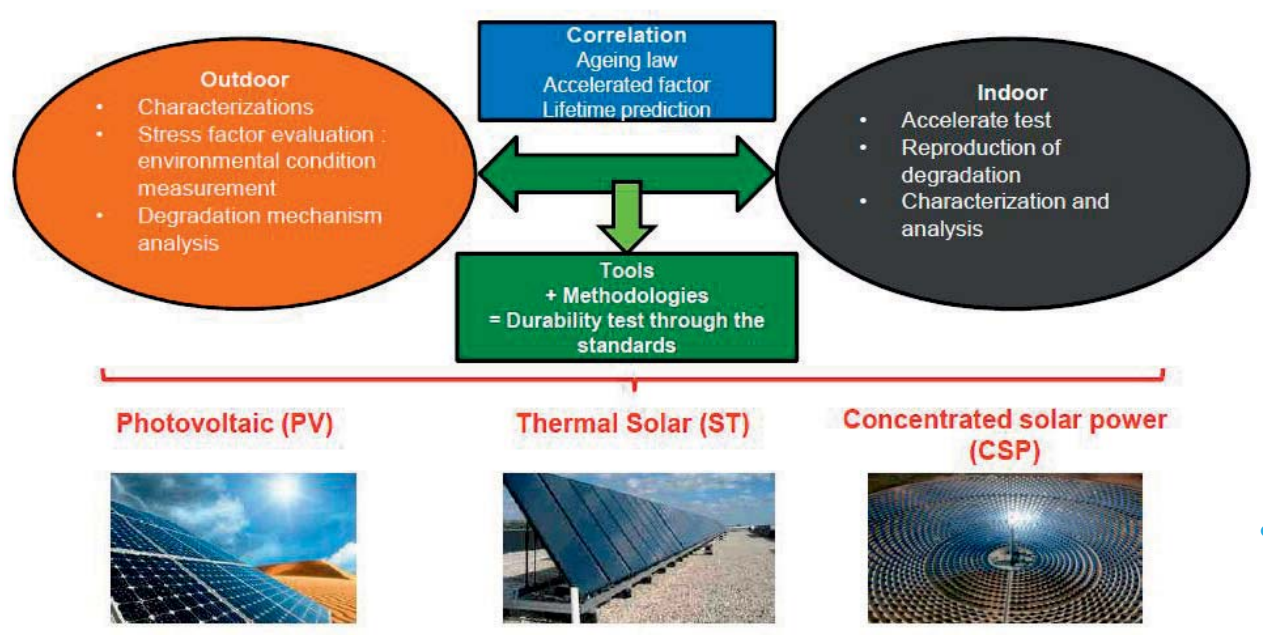

$\Delta$ Figure 1. Plateforme pour l'étude de la durabilité des matériaux et des systèmes solaires avec un réseau d'expertise et multisites pour le test extérieurs et intérieurs.

solaires : le photovoltaïque, le solaire thermique et le solaire de concentration (Fig. 1). Les sites extérieurs proposent plusieurs climats représentatifs de contraintes spécifiques et de conditions de sévérité suffisantes. Les sites intérieurs permettront de simuler ces mêmes contraintes dans des conditions accélérées et ultra-accélérées.

Durasol est un investissement de $12 \mathrm{M} €$ dont $6 \mathrm{M} €$ sont financés par le gouvernement français en tant que «Équipement d'excellence » dans le cadre des «Investissements d'avenir». Le projet va permettre de mieux comprendre les propriétés des matériaux et procédés et va permettre par des analyses physico-chimiques et des tests de méthodologie adoptée pour ce projet est dérivée de celle proposée par la tâche IEA-SHC 39 sur les matériaux polymères pour la technologie solaire.

Les apports du projet :

\section{- Pour le citoyen}

Le solaire est désormais devenu un bien de consommation. Etudier les mécanismes de vieillissement permet in fine aux industriels vendeurs de pouvoir apporter des garanties de durée de vie pour leurs produits. Cela conduit à offrir un avantage certain au citoyen-client dans ses choix et garantir des coûts d'utilisation réduits ainsi qu'une plus grande facilité d'utilisation.
- Pour le système de recherche Ce domaine est relativement peu étudié car complexe. Bien que d'autres organismes de recherche travaillent sur les études de vieillissement : le NREL aux Etats-Unis, un Fraunhofer à Karlsruhe, et un autre au Japon (institut AIST), DURASOL est le seul à s'intéresser à la compréhension fine des mécanismes de vieillissement des matériaux et technologies et à s'intéresser au solaire dans sa globalité. Cette plateforme couvrant l'ensemble des technologies solaires sera unique en Europe et va permettre à la France de consolider sa position dans le domaine des énergies renouvelables.

\section{- Pour l'économie}

Le bénéfice de ce projet est de fournir des outils et des méthodes de test aux industries du solaire afin de promouvoir de nouveaux standards de design pour concevoir les produits de demain, dans des domaines qui ne se limitent pas au photovoltaïque.

Site de la plateforme :

www.durasol.fr

\section{Coordinateur}

\section{CEA-INES}

\section{Partenaires du projet}

EDF R\&D,

INSA Lyon, LaMCoS, UMR CNRS 5259,

Université de Corse, SPE, UMR CNRS 6134

Université de Aix-Marseille, IM2NP,

UMR CNRS 7334,

Université de Clermont-Ferrand, ICCF, UMR

CNRS 6296,

Université de la Réunion, PIMENT 